\title{
Higgs-Higgsino-gaugino induced two loop electric dipole moments
}

\author{
Yingchuan Li, ${ }^{1, *}$ Stefano Profumo, ${ }^{2,+}$ and Michael Ramsey-Musolf ${ }^{1,3, *}$ \\ ${ }^{1}$ Department of Physics, University of Wisconsin, Madison, Wisconsin 53706 USA \\ ${ }^{2}$ Department of Physics and Santa Cruz. Institute for Particle Physics, University of California, 1156 High Street, Santa Cruz, \\ California 95064, USA \\ ${ }^{3}$ Kellogg Radiation Laboratory, California Institute of Technology, Pasadena, California 91125 USA
}

(Received 9 July 2008; revised manuscript received 4 September 2008; published 10 October 2008)

We compute the complete set of Higgs-mediated chargino-neutralino two-loop contributions to the electric dipole moments of the electron and neutron in the minimal supersymmetric standard model (MSSM). We study the dependence of these contributions on the parameters that govern $C P$-violation in the MSSM gauge-gaugino-Higgs-Higgsino sector. We find that contributions mediated by the exchange of $W H^{ \pm}$and $Z A^{0}$ pairs, where $H^{ \pm}$and $A^{0}$ are the charged and $C P$-odd Higgs scalars, respectively, are comparable to or dominate over those mediated by the exchange of neutral gauge bosons and $C P$-even Higgs scalars. We also emphasize that the result of this complete set of diagrams is essential for the full quantitative study of a number of phenomenological issues, such as electric dipole moment searches and their implications for electroweak baryogenesis.

DOI: 10.1103/PhysRevD.78.075009

PACS numbers: 11.30.Pb, 11.30.Er, 12.60.Jv, 13.40.Em

\section{INTRODUCTION}

The search for $C P$ violation (CPV) beyond that of the standard model (SM) lies at the forefront of nuclear and particle physics. Perhaps the most powerful probes for new CPV are searches for permanent electric dipole moments (EDMs) of the electron, neutron, and neutral atoms. Null results obtained from these searches have placed stringent constraints on CPV in the strong sector of the SM, while present and expected future sensitivities lie several orders of magnitude away from expectations based on CPV associated with the phase of the Cabibbo-Kobayashi-Maskawa (CKM) matrix. Various scenarios for CPV connected to new physics at or above the electroweak scale naturally imply the existence of nonvanishing EDMs that could be observed in future experiments. Thus, a comprehensive program of EDM searches could uncover either CPV associated with the " $\theta$ term" of the QCD Lagrangian, new electroweak scale physics, or both. Each possibility has potentially significant consequences for cosmology. The Peccei-Quinn mechanism proposed to explain the vanishingly small value of $\bar{\theta}$ implies the existence of an axion that could account for the cold dark matter, while new electroweak scale CPV could help in explaining the observed abundance of baryonic matter through the mechanism of electroweak baryogenesis (EWB).

Among the most theoretically attractive possibilities for new physics is supersymmetry (SUSY). SUSY provides an appealing solution to the naturalness problem of the SM. However, SUSY has to be softly broken to be consistent with experimental observations. In order to solve the naturalness problem, the SUSY breaking scale should be not

\footnotetext{
*yli@physics.umd.edu

+profumo@scipp.ucsc.edu

*mjrm@physics.wisc.edu
}

much higher than a few TeV. While the exact mechanism of soft SUSY breaking is not yet known, its effect is encoded into the soft terms in the low-energy realization of this scenario. In the minimal supersymmetric standard model (MSSM), the presence of soft terms implies the existence of 40 additional CPV phases beyond the single phase of the CKM matrix in the SM. As there exists no known a priori reason for these phases to be suppressed, one expects rather sizable EDMs to be generated by oneloop graphs when supersymmetric particle masses are below $\sim 1 \mathrm{TeV}$.

However, the current experiment bounds on electron, neutron EDM, and ${ }^{199} \mathrm{Hg}$ atom are already tight: $\left|d_{e}\right|<$ $1.6 \times 10^{-27} e \mathrm{~cm}\left(90 \%\right.$ C.L.) [1], $\left|d_{n}\right|<2.9 \times 10^{-26} e \mathrm{~cm}$ (90\% C.L.) [2], and $\left|d_{A}\left({ }^{199} \mathrm{Hg}\right)\right|<2.1 \times 10^{-28} e \mathrm{~cm}(95 \%$ C.L.) [3] (For recent reviews of EDM searches and their implications for SUSY, see, e.g. Refs. [4,5]). These results imply CPV phases of order $10^{-3}$ or smaller, leading to the so-called "SUSY CP problem." Its resolution, as well as that of the related "SUSY flavor problem," requires some other mechanism for suppressing one-loop EDMs (and one-loop flavor changing neutral currents). One possibility is to take the masses of the first and second generation sfermions to be of order $10 \mathrm{TeV}$ [6]. In such circumstances, the one-loop contributions to EDMs are highly suppressed, and the two-loop contributions to EDM, with $C P$ violation from either chargino-neutralino sector or the third generation of squarks, may give competitive and even dominate contributions to EDMs of the electron and neutron. ${ }^{1}$

Previous work has considered a subset of these two-loop contributions, including those involving third generation

\footnotetext{
${ }^{1}$ The EDMs of diamagnetic atoms such as ${ }^{199} \mathrm{Hg}$ will be suppressed in this limit, as they are generated primarily by the one-loop chromo-EDM operators.
} 
squarks $[7,8]$ and charginos [9-12] whose CPV interactions with the gauge-Higgs sector of the MSSM induce an EDM (or chromo-EDM) of an elementary, first generation SM fermion. In particular, implications for $C P$ violation at high-energy colliders and dominant higher-loop contributions were discussed in detail in Ref. [10]. The $C P$ violation from chargino $\left(\chi^{+}\right)$-neutralino $\left(\chi^{0}\right)$ sector can be propagated to the SM fermion though purely gauge boson exchanges. In this case, it has been shown that no $C P$ violation can be propagated though $\gamma \gamma, \gamma Z$, and $Z Z$ exchanges [11], leaving the $W W$ exchange as the only possibility. This contribution was recently calculated in Refs. [11,12]. $C P$ violation can also be propagated through the exchange of gauge and Higgs boson pairs, including $\gamma h^{0}, \gamma H^{0}, Z h^{0}, Z H^{0}, \gamma A^{0}, Z A^{0}$, and $W H^{ \pm}$. Here, $h^{0}$ and $H^{0}$ denote the neutral, $C P$-even Higgs scalars of the MSSM, with $h^{0}$ being the lightest, "SM-like" scalar; $A^{0}$ is the neutral $C P$-odd scalar; and $H^{ \pm}$denotes the charged scalars. The contributions due to $\gamma h^{0}, \gamma H^{0}, Z h^{0}, Z H^{0}$, and $\gamma A^{0}$ exchanges have been studied [9-12].

In what follows, we compute the remaining two-loop contributions that survive in the limit of large sfermion masses: Barr-Zee [13] type amplitudes wherein charginoneutralino loops communicate $\mathrm{CPV}$ to the fermion via the exchange of a $Z A^{0}$ or $W H^{ \pm}$pair. We also compute the $\gamma h^{0}$, $\gamma H^{0}, Z h^{0}, Z H^{0}$, and $\gamma A^{0}$ contributions, and compare our results with the previous computations reported in Refs. [9-12]. We report agreement with all previous results. We find that, in general, the new contributions are comparable in magnitude to those previously computed, or they are even dominant. The $Z A^{0}$ contribution is accidentally suppressed in the case of the electron EDM by the $1-$ $4 \sin ^{2} \theta_{W}$ factor, but it is important for the neutron EDM. Unlike the case of two-loop diagrams with $C P$ violation from squarks, where it has been noted that the $\gamma h^{0}$ and $\gamma A^{0}$ contributions dominate $[7,8]$, we find-after completing a numerical study of the analytic results-that the $W H^{ \pm}$ contribution is among the dominant ones for the electron $\mathrm{EDM}$, and both the $Z$ plus $H^{0}, A^{0}$ and the $W H^{ \pm}$contributions are the dominant ones for the neutron EDM, proving that the inclusion of these contributions is indispensable.

Apart from the implications for EDM phenomenology, our results also have interesting consequences for the viability of supersymmetric EWB. Indeed, part of our original motivation for computing the loops containing the $A^{0}$ and $H^{ \pm}$is that the masses of these scalars affects the dynamics of supersymmetric EWB. In particular, during a first-order electroweak phase transition that proceeds via bubble nucleation, the rate at which the neutral Higgs vacuum expectation values change across the bubble walls depends on $m_{A^{0}}$ (which also sets the scale for $m_{H^{ \pm}}$). Since the $C P$-violating asymmetries needed for baryon number production are generated during the phase transition by scattering from these vacuum expectation values, knowledge of the bubble wall profiles and their dependence on the other MSSM parameters is essential for determining the viability of supersymmetric EWB. In general, SUSY EWB is enhanced for relatively light $m_{A^{0}}$-a region in which the corresponding $Z A^{0}, Z H^{0} \gamma A^{0}, \gamma H^{0}$, and $W H^{ \pm}$ EDM contributions are also enhanced. In our numerical study of the two-loop EDMs, we investigate the corresponding $m_{A^{0}}$ dependence with an eye to these implications for EWB.

Our discussion of these points is organized in the remainder of the paper as follows. In Sec. II we provide details of our two-loop computation and the analytic expressions for the results. Section III gives our numerical analysis. We summarize our results in Sec. IV, while additional technical details are provided in the appendix. We note that, during the course of completing our study, a parallel computation of the two-loop EDMs in SUSY using an effective field theory approach also appeared [14]. We comment on the similarities and differences we find with that analysis.

\section{TWO-LOOP EDMS: HIGGS-GAUGE MEDIATED $\chi^{+}-\chi^{0}$ CONTRIBUTIONS}

Representative diagrams from the various topologies we consider are shown in Fig. 1. For simplicity we have not shown the different crossed graphs or those in which scalar and vector boson lines are interchanged. In addition, diagrams involving a photon insertion on the SM fermion line are also not shown, as these contributions vanish. Of the remaining, nonvanishing diagrams, the contributions with neutral Higgs boson exchange, namely, $\gamma h^{0}, \gamma H^{0}, Z h^{0}$, $Z H^{0}$, and $\gamma A^{0}, Z A^{0}$, only involve diagram (a), while the contributions with charged Higgs boson exchange $W^{\mp} H^{ \pm}$ involve all the diagrams (a, b, c, d). To simplify the

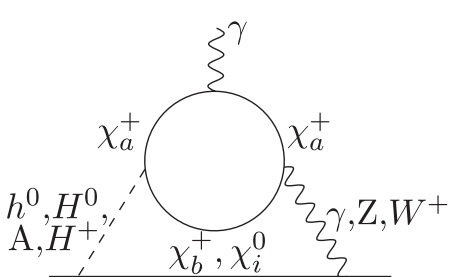

(a)

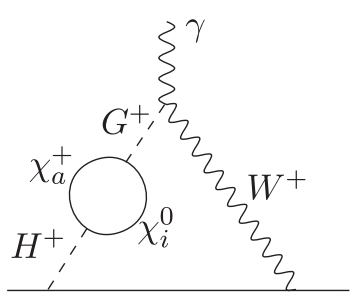

(c)

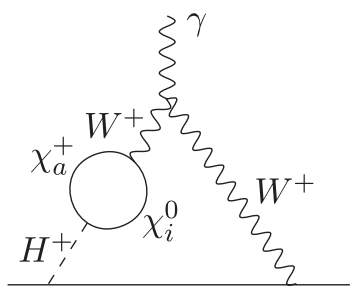

(b)

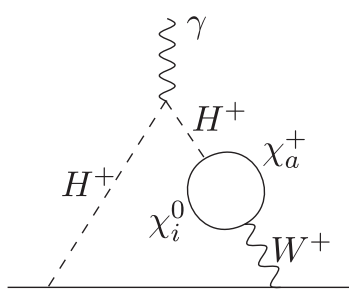

(d)
FIG. 1. All the two-loop diagrams with chargino-neutralino loop mediated by Higgs bosons. (Mirror graphs are not displayed.) 
computation of the latter, we follow Ref. [8] and use the nonlinear $R_{\xi}$ gauge [15]. The corresponding gauge-fixing term in the Lagrangian is obtained by replacing the ordinary derivative that appears in the $R_{\xi}$ gauge

$$
\mathcal{L}_{\text {g.f. }}^{R_{\xi}}=-\frac{1}{2 \xi}\left|\partial_{\mu} W_{\mu}^{+}-i \xi M_{W} \phi^{+}\right|^{2},
$$

with the $\mathrm{U}(1)_{\mathrm{EM}}$ covariant derivative $D_{\mu}=\partial_{\mu}-i e A_{\mu}$ in nonlinear $R_{\xi}$ gauge

$$
\mathcal{L}_{\text {g.f. }}^{\text {nonlinear } R_{\xi}}=-\frac{1}{2 \xi}\left|D_{\mu} W_{\mu}^{+}-i \xi M_{W} \phi^{+}\right|^{2} .
$$

Thus, just as the $R_{\xi}$ gauge is designed to eliminate mixing between the would-be Goldstone boson $G^{ \pm}$and the $W^{ \pm}$ implied by the Higgs kinetic term $\left(D_{\mu} \phi\right)^{+}\left(D^{\mu} \phi\right)$, the nonlinear $R_{\xi}$ gauge is constructed in such a way that the coupling $G^{ \pm} W^{\mp} \gamma$ arising from the same kinetic term is also canceled. As a result, the $W^{+} W^{-} \gamma$ vertex is modified from its standard form in the conventional renormalizable gauges.

A direct-and simplifying — consequence of employing the nonlinear $R_{\xi}$ gauge is that the contribution from diagram (c) vanishes due to the absence of the $G^{ \pm} W^{\mp} \gamma$ coupling. Moreover, an additional simplification can be obtained when carrying out the computation in the Landau gauge $(\xi \rightarrow 0)$. In doing so, one must take care to first compute the $\xi \neq 0$ contributions to the $W^{+} W^{-} \gamma$ vertex and $W$-boson propagators in Fig. 1(b) and carry out the appropriate contractions that appear in the one-loop amplitude before taking the $\xi \rightarrow 0$ limit, since the additional contribution to the $W^{+} W^{-} \gamma$ vertex arising in the nonlinear $R_{\xi}$ gauge is proportional to $1 / \xi$. The resulting simplification is that the amplitude from Fig. 1(d) vanishes as well. This is because the chargino-neutralino loop in (d) is proportional to the four momentum of the $W$ boson, while the propagator of $W$ is transverse in Landau gauge.

In carrying out the calculation, we first compute out the one-loop subgraphs corresponding to the amplitude for $\gamma(q, \mu) \rightarrow h(q-\ell)+g(\ell, \nu)$, where $h$ stands for one of Higgs bosons $\left(h^{0}, H^{0}, A^{0}, H^{ \pm}\right)$having momentum $q-\ell ; g$ denotes one of gauge bosons $(\gamma, Z, W)$ having momentum $\ell$; and $\mu$ and $\nu$ denote the vector indices associated with the external photon and $g$, respectively. Gauge invariance implies that the amplitude involves a linear combination of the (pseudo)tensors

$$
P^{\mu \nu}=\epsilon^{\mu \nu \alpha \beta} q_{\alpha} \ell_{\beta}, \quad T^{\mu \nu}=\ell^{\mu} q^{\nu}-g^{\mu \nu} \ell \cdot q .
$$

For the full two-loop graphs involving the exchange of neutral bosons, only $P^{\mu \nu}$ contributes in the case of $C P$-even Higgs exchange, while only $T^{\mu \nu}$ contributes for the graphs involving the $C P$-odd Higgs. Both $P^{\mu \nu}$ and $T^{\mu \nu}$ contribute to the two-loop $W H^{ \pm}$amplitude. For our particular gauge choice, we find that $P^{\mu \nu}$ arises from Fig. 1(a) alone, while for $W^{\mp} H^{ \pm}$exchange, $T^{\mu \nu}$ requires the sum of both Figs. 1(a) and 1(b) [graph (b) only generates a $\ell$. $q g^{\mu \nu}$ structure]. We will use these features to explain the origin of the overall, relative signs between the various contributions below.

In obtaining our final results for the two-loop contributions, we use the Feynman rules and conventions given in Ref. [16]. ${ }^{2}$ We have attempted to express our results in a manner that makes it easy to directly compare with the earlier work of Refs. [9-12]. We find

$$
\begin{aligned}
d_{f}^{\gamma S}= & \frac{e Q_{f} \alpha^{2} c_{f}^{S}}{8 \sqrt{2} \pi^{2} s_{W}^{2}} \frac{m_{f}}{M_{W} m_{S}^{2}} \\
& \times \sum_{a=1}^{2} \operatorname{Im}\left(D_{S, a a}^{R}\right) M_{\chi_{a}^{+}} \int_{0}^{1} d x \frac{1}{x(1-x)} j\left(0, \frac{r_{a S}}{x(1-x)}\right),
\end{aligned}
$$

$$
\begin{aligned}
d_{f}^{Z S}= & \frac{e \alpha^{2}\left(T_{3 f_{L}}-2 s_{W}^{2} Q_{f}\right) c_{f}^{S}}{16 \sqrt{2} \pi^{2} c_{W}^{2} s_{W}^{4}} \frac{m_{f}}{M_{W} m_{S}^{2}} \sum_{a, b=1}^{2} \operatorname{Im}\left(G_{a b}^{R} D_{S, b a}^{R}\right. \\
& \left.-G_{a b}^{L} D_{S, b a}^{L}\right) M_{\chi_{b}^{+}} \int_{0}^{1} d x \frac{1}{x} j\left(r_{Z S}, \frac{x r_{a S}+(1-x) r_{b S}}{x(1-x)}\right),
\end{aligned}
$$

$$
\begin{aligned}
d_{f}^{\gamma A^{0}}= & \frac{e Q_{f} \alpha^{2} c_{f}^{A^{0}}}{8 \sqrt{2} \pi^{2} s_{W}^{2}} \frac{m_{f}}{M_{W} m_{A^{0}}^{2}} \\
& \times \sum_{a=1}^{2} \operatorname{Im} E_{a a}^{R} M_{\chi_{a}^{+}} \int_{0}^{1} d x \frac{1-2 x+2 x^{2}}{x(1-x)} j\left(0, \frac{r_{a A^{0}}}{x(1-x)}\right),
\end{aligned}
$$

$$
\begin{aligned}
d_{f}^{Z A^{0}}= & \frac{e \alpha^{2}\left(T_{3 f_{L}}-2 s_{W}^{2} Q_{f}\right) c_{f}^{A^{0}}}{16 \sqrt{2} \pi^{2} c_{W}^{2} s_{W}^{4}} \frac{m_{f}}{M_{W} m_{A^{0}}^{2}} \\
& \times \sum_{a, b=1}^{2} \operatorname{Im}\left(G_{a b}^{R} E_{b a}^{R}+G_{a b}^{L} E_{b a}^{L}\right) M_{\chi_{b}^{+}} \\
& \times \int_{0}^{1} d x \frac{1-x}{x} j\left(r_{Z A^{0}}, \frac{x r_{a A^{0}}+(1-x) r_{b A^{0}}}{x(1-x)}\right),
\end{aligned}
$$

$$
\begin{aligned}
d_{f}^{W H^{ \pm}}= & -\frac{e \alpha^{2} c_{f}^{H^{+}}}{32 \pi^{2} s_{W}^{4} c_{W}} \frac{m_{f}}{M_{W} m_{H^{+}}^{2}} \sum_{a=1}^{2} \sum_{i=1}^{4} \int_{0}^{1} d x \\
& \times \frac{1}{1-x} j\left(r_{W H^{+}}, \frac{r_{a H^{+}}}{1-x}+\frac{r_{i H^{+}}}{x}\right)\left[\left(\operatorname { I m } \left(M_{a i}^{L} N_{a i}^{L *}\right.\right.\right. \\
& \left.+M_{a i}^{R} N_{a i}^{R *}\right) M_{\chi_{a}^{+}} x^{2}+\operatorname{Im}\left(M_{a i}^{R} N_{a i}^{L *}+M_{a i}^{L} N_{a i}^{R *}\right) M_{\chi_{i}^{0}} \\
& \left.\times(1-x)^{2}\right)+\left(\operatorname{Im}\left(M_{a i}^{L} N_{a i}^{L *}-M_{a i}^{R} N_{a i}^{R *}\right) M_{\chi_{a}^{+}} x\right. \\
& \left.\left.+\operatorname{Im}\left(M_{a i}^{R} N_{a i}^{L *}-M_{a i}^{L} N_{a i}^{R *}\right) M_{\chi_{i}^{0}}(1-x)\right)\right] .
\end{aligned}
$$

\footnotetext{
${ }^{2}$ However, our convention for the Higgs scalar mixing angle $\alpha$ differs from that of Ref. [16]. To facilitate comparison with results appearing in the literature, we adopt the convention of Ref. [12].
} 
Here, $s_{W}=\sin \theta_{W}$ and $c_{W}=\cos \theta_{W}$. The $S$ in Eq. (4) and (5) denotes $h^{0}$ and $H^{0}$. The symbol $f=u, d$, e represents the up quark, down quark, and electron, respectively; $Q_{f}$ and $m_{f}$ are the electric charge and mass, respectively, of fermion $f$, and $T_{3 f_{L}}$ is the third component of the weak isospin of its left-handed component; finally, $j\left(r, r^{\prime}\right)$ is the loop function defined in Ref. [11] and given in the appendix.

The mass ratios in loop functions are $r_{Z h^{0}}=M_{Z}^{2} / m_{h^{0}}^{2}$, $r_{Z H^{0}}=M_{Z}^{2} / m_{H^{0}}^{2}, \quad r_{Z A^{0}}=M_{Z}^{2} / m_{A^{0}}^{2}, \quad r_{a h^{0}}=M_{\chi_{a}^{+}}^{2} / m_{h^{0}}^{2}$, $r_{a H^{0}}=M_{\chi_{a}^{+}}^{2} / m_{H^{0}}^{2}, \quad r_{a A^{0}}=M_{\chi_{a}^{+}}^{2} / m_{A^{0}}^{2}, r_{W H^{+}}=M_{W}^{2} / m_{H^{+}}^{2}$, $r_{a H^{+}}=M_{\chi_{a}^{+}}^{2} / m_{H^{+}}^{2}$, and $r_{i H^{+}}=M_{\chi_{i}^{0}}^{2} / m_{H^{+}}^{2}$, with $M_{W, Z}$, the masses of $W$ and $Z$ gauge bosons, $m_{h^{0}, H^{0}, A^{0}, H^{+}}$, the masses of Higgs bosons, and $M_{\chi_{a}^{+}} \geq 0$ and $M_{\chi_{i}^{0}} \geq 0$, the masses of charginos and neutralinos, respectively. The coefficients $c_{u, d, e}^{h^{0}, H^{0}, A^{0}, H^{+}}$and matrices $D_{h^{0}, H^{0}}^{R, L}, G^{R, L}, E^{R, L}$, $M^{R, L}, N^{R, L}$ involve various combinations of the chargino and neutralino couplings to Higgs and gauge bosons, and are collected explicitly in the appendix.

Before proceeding with our numerical study, we make several comments on the analytic results.

(i) The dependence on the CPV phases in the gaugegaugino-Higgs-Higgsino sector is contained in the imaginary parts of the couplings $D_{S, a a}^{R}$, etc. but not separated out explicitly. As indicated in the appendix, these phases arise from diagonalizing the chargino and neutralino mass matrices in Eq. (A3). In general, the resulting independent phases are $\operatorname{Arg}\left(\mu M_{i} b^{*}\right)$ and $\operatorname{Arg}\left(M_{i} M_{j}^{*}\right)$, where $\mu$ is the supersymmetric Higgs-Higgsino mass parameter; $M_{i}(i=$ $1,2,3)$ are the soft gaugino mass parameters; and $b$ is the soft Higgs mass parameter. The $\mathrm{SU}(3)_{C}$ mass parameter does not enter the diagonalization of chargino-neutralino mass matrices, leaving two remaining phases. The analysis of CPV in this sector is often simplified by assuming that $\operatorname{Arg}\left(M_{1} M_{2}^{*}\right)=0$, leaving one remaining, independent phase denoted $\phi_{\mu}$. In our numerical study below, we will adopt this simplifying assumption and verify numerically that each of the two-loop contributions is proportional to $\sin \phi_{\mu}$. We also comment on the impact of relaxing this assumption.

(ii) The coefficients $c_{f}^{h^{0}}$ and $c_{f}^{H^{0}}$ given in Eq. (A1) as well as the matrices $D_{h^{0}}^{R, L}$ etc. given in Eq. (A2) depend in general on $\tan \beta=v_{u} / v_{d}$, where the $v_{k}$ are the vacuum expectation values of the two neutral Higgs scalars, and $Z_{R}$, which further depends on $m_{A^{0}}$ as illustrated in Eq. (A6). This introduces an additional dependence on $\tan \beta$ and $m_{A^{0}}$ beyond the explicit dependence generated by the Yukawa couplings and dependence of the loop functions on scalar masses.

(iii) The overall sign in the expression for the $W H^{ \pm}$ contribution is opposite to that of the other contribu- tions. The origin of this overall sign can be understood by considering the combinations of couplings and Lorentz structures entering the two-loop amplitudes. To illustrate, we consider the loop of Fig. 1(a) that enters each of the contributions. To compare the relative signs of the couplings, we define a general set of interactions involving charginos, neutralinos, Higgs scalars, and fermions:

$$
\begin{aligned}
\mathcal{L}_{V \chi \chi} & =\bar{\chi} \gamma^{\mu}\left[A_{L} P_{L}+A_{R} P_{R}\right] \chi V_{\mu}+\cdots, \\
\mathcal{L}_{\phi \chi \chi} & =\bar{\chi}\left[B_{L} P_{L}+B_{R} P_{R}\right] \chi \phi+\cdots, \\
\mathcal{L}_{V \ell \ell} & =\bar{\ell} \gamma^{\mu}\left[C_{L} P_{L}+C_{R} P_{R}\right] \ell V_{\mu}+\cdots, \\
\mathcal{L}_{\phi \ell \ell} & =\bar{\ell}\left[D_{L} P_{L}+D_{R} P_{R}\right] \ell \phi+\cdots .
\end{aligned}
$$

In Table I below we give the corresponding phases of the couplings obtained from the Feynman rules of Ref. [16]. Now we consider the structure of the fermion line in the loop, which gives the only other source of a phase difference between the different contributions. If $\ell$ is the momentum flowing through the loop (we may neglect the external fermion momenta for this discussion), we have

$$
\begin{aligned}
Z A^{0} & \sim \gamma^{\mu}\left[2 T_{3} P_{L}-2 Q \sin ^{2} \theta_{W}\right] \ell \gamma_{5} \\
& =\gamma^{\mu}\left[-2 T_{3} P_{L}+2 Q \sin ^{2} \theta_{W} \gamma_{5}\right] \ell \\
& =\frac{1}{2} \gamma^{\mu}\left[-\left(2 T_{3}-4 Q \sin ^{2} \theta_{W}\right) \gamma_{5}-2 T_{3}\right] \ell, \\
Z h^{0} & \sim \gamma^{\mu}\left[2 T_{3} P_{L}-2 Q \sin ^{2} \theta_{W}\right] \ell \\
& =\frac{1}{2} \gamma^{\mu}\left[\left(2 T_{3}-4 Q \sin ^{2} \theta_{W}\right)-2 T_{3} \gamma_{5}\right] \ell, \\
W H^{ \pm} & \sim \gamma^{\mu} P_{L} \ell P_{R}=\gamma^{\mu} P_{L} \ell=\frac{1}{2} \gamma^{\mu}\left(1-\gamma_{5}\right) \ell .(10)
\end{aligned}
$$

For the case of $Z A^{0}$ exchange which involves $T^{\mu \nu}$ from the closed chargino loop, we require the $\gamma_{5}$ term from the lower line to obtain the EDM, whereas for $Z h^{0}$ exchange, we have the pseudotensor $P^{\mu \nu}$ from the closed chargino loop, necessitating that we retain the identity matrix term from the fermion line. For the $W H^{ \pm}$exchange contribution, we require both. Table II gives the resulting overall phase for the various contributions.

We observe that the expression for the part of the $W H^{ \pm}$exchange graph arising from the $T^{\mu \nu}$ tensor should have an opposite, overall phase compared to

TABLE I. Phases of couplings in Eq. (9) as they enter the amplitude of Fig. 1(a).

\begin{tabular}{lccr}
\hline \hline Vertex & $Z A$ & $Z h^{0}$ & $W H^{ \pm}$ \\
\hline$V \chi \chi$ & $-i$ & $-i$ & $+i$ \\
$\phi \chi \chi$ & +1 & $-i$ & $+i$ \\
$\phi \ell \ell$ & +1 & $+i$ & $-i$ \\
$V \ell \ell$ & $+i$ & $+i$ & $+i$ \\
Overall & +1 & +1 & -1 \\
\hline \hline
\end{tabular}


TABLE II. Summary of signs from fermion line and overall result. The final row is obtained by multiplying the overall phases from Table I and the sign obtained from the fermion line.

\begin{tabular}{lcccc}
\hline \hline Graph & $Z A\left(T^{\mu \nu}\right)$ & $Z h^{0}\left(P^{\mu \nu}\right)$ & $W H^{ \pm}\left(T^{\mu \nu}\right)$ & $W H^{ \pm}\left(P^{\mu \nu}\right)$ \\
\hline$\gamma$-matrix & $\gamma_{5}$ & 1 & $\gamma_{5}$ & 1 \\
sign & -1 & +1 & -1 & +1 \\
overall & -1 & +1 & +1 & -1 \\
\hline \hline
\end{tabular}

the corresponding term for the $Z A$ exchange diagram. Similarly, the $P^{\mu \nu}$ component of the $W H^{ \pm}$ loop and the $Z h^{0}$ graph will also differ in overall relative phase. Note that there is an additional overall phase that arises between the $T^{\mu \nu}$ and $P^{\mu \nu}$ terms when the identity

$$
\varepsilon^{\mu \nu \alpha \beta} \sigma_{\alpha \beta}=-2 i \sigma^{\mu \nu} \gamma_{5}
$$

is used in the terms generated by $P^{\mu \nu}$. Thus, the relative sign between the $Z A^{0}$ and $Z h^{0}$ graphs are the same, as are the relative phase between the $T^{\mu \nu}$ and $P^{\mu \nu}$ terms in the $W H^{ \pm}$contribution. As we discuss below, the resulting overall sign in Eq. (8) is compensated by the signs of various matrix element combinations $M_{a i}^{L} N_{a i}^{L *}$ etc. that enter the sum over chargino-neutralino intermediate states in the $\tan \beta \geqslant 1$ regime. Consequently, the $W H^{ \pm}$and other gauge boson-scalar exchange contributions to the electron and down-quark EDMs add coherently in the phenomenologically allowed regions of MSSM parameter space.

(iv) Our results for the $\gamma h^{0}, \gamma A^{0}$, and $Z h^{0}$ amplitudes agree with those of Refs. [9-11], including the overall phase. On the surface, our result for the $Z h^{0}$ contribution appears to be different from the expression given in Ref. [11]. The difference amounts to replacing the $x^{-1}$ in Eq. (5) by $[2 x(1-x)]^{-1}$ to convert the integral to that of Ref. [11]. However, after taking into account the symmetry properties of the integrands in both expressions, we have verified (both analytically and numerically) that they agree.

(v) A direct comparison of our analytic results with those obtained in Ref. [14] is not straightforward, since the latter employed an effective field theory approach. We note, however, that these authors also include a nonzero result for the ZZ-exchange contribution that the authors of Ref. [11] argued should vanish. A direct comparison of numerical results is also challenging, since only the dependence of the EDMs on the CPV phases was given in Ref. [14], whereas in our numerical study below, we explore the dependence on mass parameters and $\tan \beta$ for fixed values of $\phi_{\mu}$.

\section{NUMERICAL ANALYSIS}

In this section we numerically assess the impact of the additional, two-loop EDM contributions discussed above. As mentioned in the introduction, one motivation for our work to consider the complete set of two-loop Higgsmediated chargino-neutralino contributions stems from the framework of EWB. It is therefore natural and well motivated to focus a portion of our numerical analysis on a supersymmetric setup which is compatible with that framework. Before doing so, however, we investigate the relative importance of the various contributions and their dependence on MSSM parameters.

To that end, we define a benchmark parameter set scenario that will serve as a basis for comparison, motivated by the EWB framework, and consistent with phenomenological and cosmological constraints, as discussed below. We then proceed with a scan over pairs of parameters that govern the size of EDMs, keeping the other parameters fixed at their benchmark values. To suppress one-loop EDM contributions, we assume all sfermions to be decoupled (we set all sfermion soft breaking masses to $10 \mathrm{TeV}$, and the trilinear scalar couplings to zero, for definiteness). The gluino mass is entirely unimportant for the phenomenology discussed here, and is set to $1 \mathrm{TeV}$. The remaining parameters relevant for the two-loop EDM contributions are the absolute values of the gaugino soft breaking masses $M_{1,2}$ and of the Higgsino mass parameter $\mu$, the heavy MSSM Higgs mass scale (for definiteness we employ here as a free parameter $m_{A^{0}}$ ), and $\tan \beta$. Our reference benchmark setup is defined as follows:

$$
\begin{gathered}
M_{1}=145 \mathrm{GeV}, \quad M_{2}=290 \mathrm{GeV}, \\
\mu=300 \mathrm{GeV}, \quad m_{A^{0}}=300 \mathrm{GeV}, \quad \tan \beta=10 .
\end{gathered}
$$

We consider here one single $C P$-violating phase, $\phi_{\mu}$, as discussed above. We set this phase $\phi_{\mu}=\pi / 2$, giving the largest $C P$-violating effect. We verified numerically that the EDMs considered here scale proportionally to $\sin \phi_{\mu}$ to within an accuracy of $1 \%$. This means that (1) all the results we show below can be simply rescaled when assuming a nonmaximal $C P$-violating phase and (2) we show the largest possible size for the EDM contributions we consider here. ${ }^{3}$ Notice that the values for $m_{h^{0}}$ and the mixing angle $\alpha$ (at the two-loop level) as well as all the supersymmetric masses and mixing are obtained numerically through the FeynHiggs package [18].

We have chosen this particular benchmark setup for several reasons. First, this choice is potentially compatible with successful EWB. Second, the lightest neutralino relic abundance is close to the observed cold dark matter density [19]. If the relic neutralino abundance were larger than the

\footnotetext{
${ }^{3}$ We remind the reader that EWB implies $\sin \phi_{\mu} \gtrsim 10^{-2}$ [17].
} 
cold dark matter density, a mechanism would be needed to dilute the relic abundance, with implications for EWB as well [20]. Third, the parameter values given above are consistent with collider searches, with precision electroweak data, including the muon anomalous magnetic moment, and with the inclusive branching ratio $b \rightarrow s \gamma$ [21]. The latter constraint is particularly critical at low $m_{A^{0}}$, since contributions to $b \rightarrow s \gamma$ from the top-quark- $H^{ \pm}$ loop can be sizable.

Starting from this reference point, we first illustrate the relative magnitude of two-loop contributions in Fig. 2. Here, we explore these contributions to the EDM of the electron (left panel) and the neutron (right panel) as a function of $\tan \beta$, with all other parameters set as in Eq. (11). To illustrate out results on a logarithmic scale, we show absolute values and indicate with black lines positive contributions and with red lines negative ones (the only negative contribution we find is from the $W W$ loop, for which we take the expression given in Ref. [11].

For the computation of the neutron EDM from the quark EDM, we employ the naïve constituent quark model relation

$$
d_{n}=\frac{4}{3} d_{d}-\frac{1}{3} d_{u} .
$$

The computation of $d_{n}$ is subject to considerable theoretical uncertainty associated with the nonperturbative strong interaction (for a discussion see, e.g., Refs. [4,22]). QCD contributions to the renormalization group evolution of the quark EDM operators from the weak scale to the hadronic

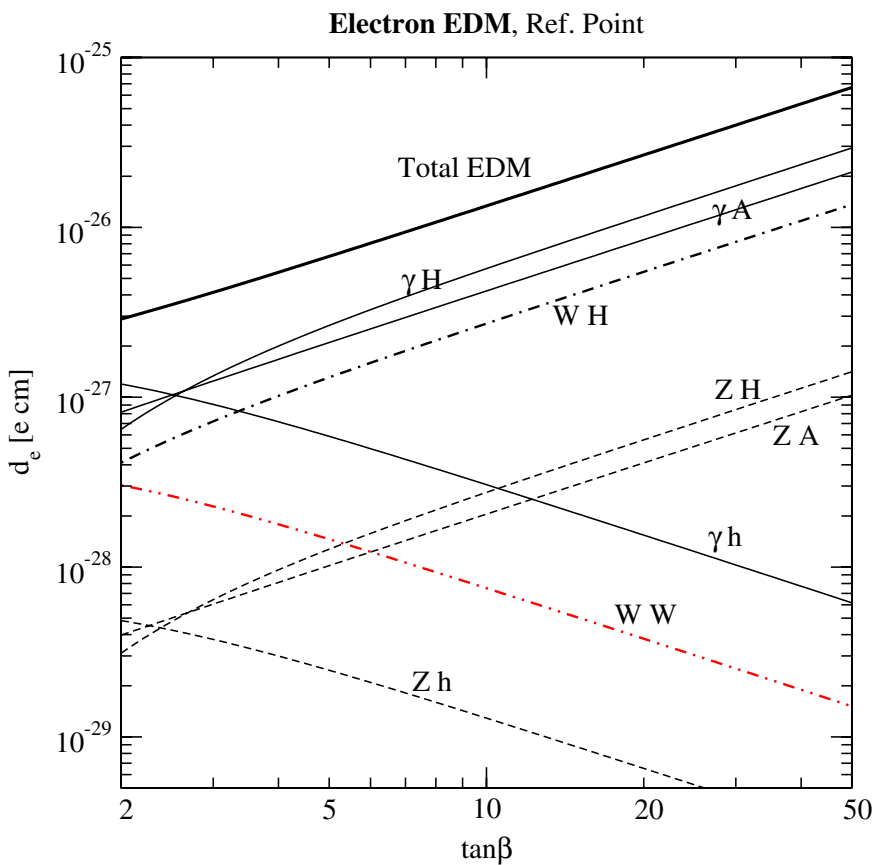

scale lead to an overall enhancement factor of 1.53 that multiplies the combination of up- and down-quark EDMs on the right side or Eq. (12) [23]. Alternately, the use of QCD sum rule techniques to compute neutron matrix elements of the quark EDM operators leads to a multiplicative enhancement factor of $1.4 \pm 0.6$ associated with the $q \bar{q}$ condensate [4]. Ellis and Flores have computed this matrix element by relating the quark EDM contributions to the corresponding quark contributions to the nucleon spin as implied by polarized deep inelastic scattering measurements, neutron $\beta$-decay, and the Bjorken sum rule [24]. This approach leads to a different weighting of the up- and down-quark contributions than appears in the Eq. (12) as well as a substantial strange quark contribution that is absent from the constituent quark model and QCD sum rule computations. In light of these variations, we consider Eq. (12) to provide a reasonable benchmark, bearing in mind that a first principles (lattice) QCD computation may yield a different dependence on the light quark EDMs.

The resulting curves in Fig. 2 lead to several observations.

(i) As indicated earlier, the sum over all intermediate chargino-neutralino states in Eq. (8) compensates for the overall relative sign in the expression for the $W H^{ \pm}$contribution. After analyzing the individual contributions in detail, we find that although the contribution from the lightest $\chi^{+}-\chi^{0}$ pair is negative (corresponding to the explicit sign in front of the expression), the sum is dominated by the remaining

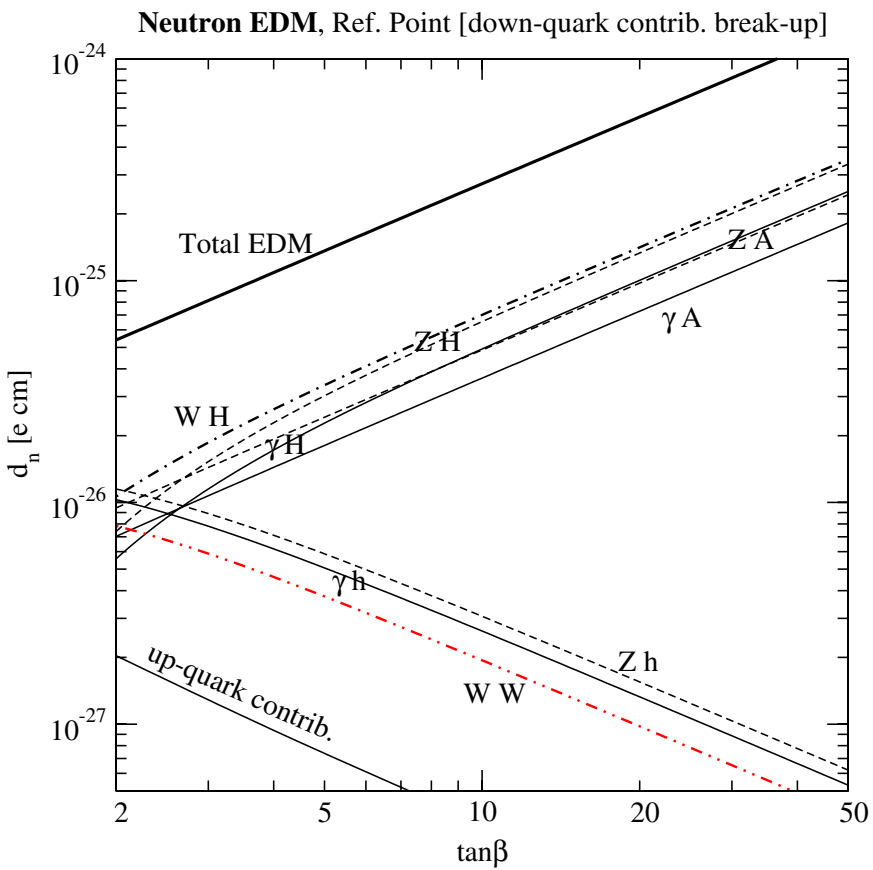

FIG. 2 (color online). A break-up of the various Higgs-mediated chargino-neutralino two-loop contributions to the electron (left) and neutron (right) electric dipole moment. Black lines correspond to positive values, red lines to negative values. In the right panel we show the various down-quark contributions times a factor $4 / 3$, as well as the global up-quark contribution (times a factor $-1 / 3$ ). All SUSY parameters, except $\tan \beta$, are fixed to the reference setup. 
sets of intermediate states, many of which carry an opposite relative sign due to the chargino-neutralino mixing matrix elements. As a result, the $W H^{ \pm}$and other gauge boson-scalar exchange contributions to the electron and down-quark EDMs carry the same relative sign.

(ii) All contributions involving heavy Higgses scale linearly with $\tan \beta$, while the $W W$ and the contributions involving $h^{0}$ scale as $1 / \tan \beta$.

(iii) The dominant contributions to the electron EDM appear to be the $\gamma H^{0}, \gamma A^{0}$, and $W H^{ \pm}$loops. However, notice that the $\gamma h^{0}$ contribution dominates at small $\tan \beta \sim 2$, and that the $W W$ contribution is also sizable in that regime. The $Z$ plus Higgs contributions are suppressed by the $T_{3 e_{L}}-2 s_{W}^{2} Q_{e}$ factor, and are relatively subdominant.

(iv) A similar picture applies to the case of the neutron EDM. Here we explicitly show only the break-up for the down-quark EDM contribution (times a factor $4 / 3$ ), and the overall up-quark contribution (times a factor $-1 / 3$ ). As opposed to the electron EDM, the $Z H^{0}, Z A^{0}$, and $W H^{ \pm}$contributions dominate the down-quark EDM.

Using the foregoing considerations, we now study the dependence of the total two-loop EDMs as a function of various MSSM parameters. First, we investigate the $\left(\tan \beta, m_{A^{0}}\right)$ sector. This sets the mass scale for all loops involving $H^{0}, A^{0}$, and $H^{ \pm}$, as well as various couplings, directly [see e.g. Eq. (A1)] or indirectly, e.g., through electroweak symmetry breaking effects in the neutralino and chargino mass and mixing matrices. We explore the

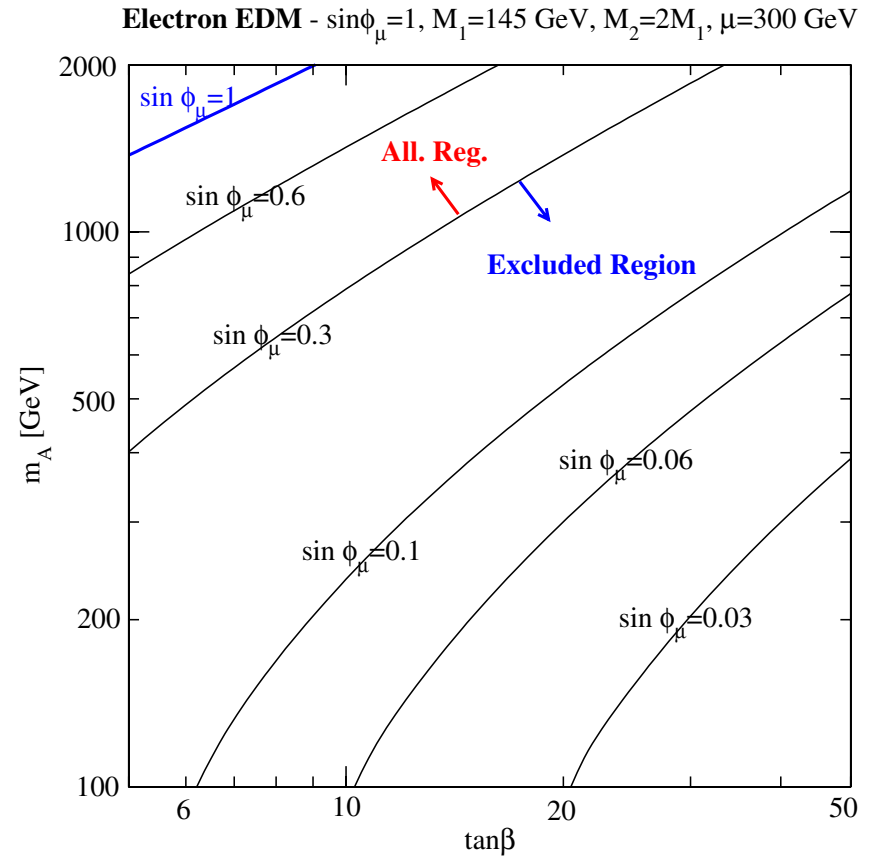

EDM dependence on $\left(\tan \beta, m_{A^{0}}\right)$ in Fig. 3, setting again all other supersymmetric parameters to the values indicated in (11). We indicate in the figure the values of the $C P$ violating phase $\sin \phi_{\mu}$ such that the resulting EDM equals the current experimental limit, $d_{e}=1.6 \times 10^{-27} e \mathrm{~cm}$ (left) and $2.9 \times 10^{-26} e \mathrm{~cm}$ (right). As expected, we find a suppression of the EDM with increasing $m_{A^{0}}$ at fixed values of $\tan \beta$, thereby allowing for consistency between experimental limits and larger values of $\left|\sin \phi_{\mu}\right|$. The overall behavior of the electron and the neutron EDM is remarkably similar. Presumably, this similarity indicates that (1) the up-quark contribution to $d_{n}$ is subdominant (see Fig. 2, right panel; we actually find that it features significant cancellations among the various contributions), and that (2) there are a few dominant contributions to $d_{e}$ and to $d_{d}$ that are simply proportional to each other, and hence they contribute in a similar fashion.

To make the connection with EWB, we now analyze the ( $\mu, M_{1,2}$ )-dependence of the two-loop EDMs. A generic expectation of the EWB scenario for the MSSM particle spectrum includes a relatively light mass scale for the heavy MSSM Higgs sector. This scenario depends on the suppression of the net baryon number density generated at the EW phase transition with $m_{A^{0}}$, as pointed out and quantified e.g. in Ref. [25]. It was also realized in several analyses (see e.g. Refs. $[17,20,26]$ and references therein), that the requirement of sufficiently large $C P$-violating sources for successful EWB prefers a resonant enhancement in the Higgsino-gaugino sources. This resonance occurs for $M_{1} \sim \mu$, the resonant neutralino baryogenesis funnel, or $M_{2} \sim \mu$, the resonant chargino baryogenesis

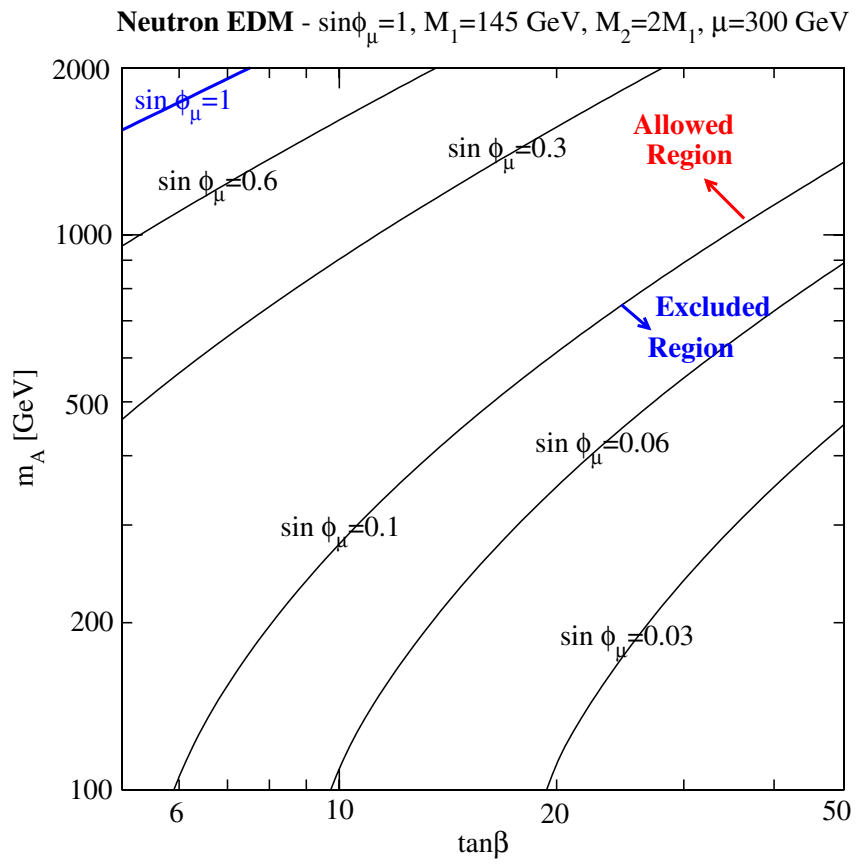

FIG. 3 (color online). Exclusion limits for the electron (left) and neutron (right) electric dipole moment, on the (tan $\left.\beta, m_{A^{0}}\right)$ plane, for $M_{1}=145 \mathrm{GeV}, M_{2}=290 \mathrm{GeV}, \mu=300 \mathrm{GeV}$ and for various values of the $C P$ violating phase $\sin \phi_{\mu}$. 
Electron EDM $-\sin \phi_{\mu}=1, M_{A}=300 \mathrm{GeV}, M_{2}=2 M_{1}[m S U G R A]$
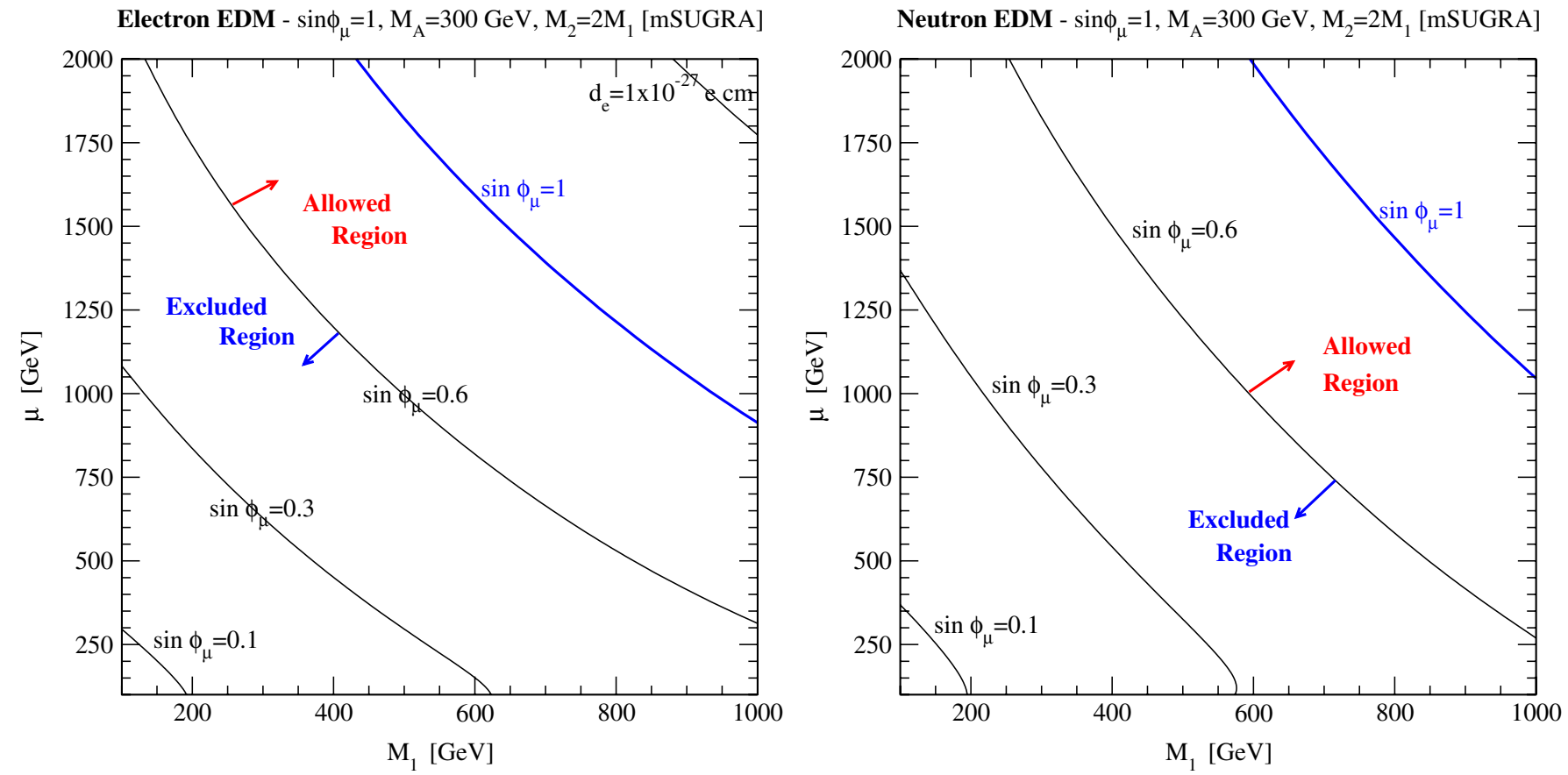

FIG. 4 (color online). Exclusion limits for the electron (left) and neutron (right) electric dipole moment, on the $\left(M_{1}, \mu\right)$ plane. We assume the gaugino unification mass relation $M_{2} \simeq 2 M_{1}$, and vary the CPV phase $\sin \phi_{\mu}$ (see the text for further details on the model assumptions). In the upper right corner of the left panel we also show the contour of electron EDM equal to $10^{-27} e \mathrm{~cm}$, for maximal CPV phase.

funnel, with $M_{1,2}, \mu \lesssim 1 \mathrm{TeV}$. An additional resonance could occur for the CPV stop sources, but the latter possibility is generally precluded by the LEP limits on the mass of the $h^{0}$. In both cases, however, successful EWB implies sub-TeV masses for Higgsinos and gauginos.

Another generic feature of the MSSM spectrum implied by successful EWB is a light, mostly right-handed stop, in order to make the EW phase transition more strongly first order. Several studies pointed out, however, that an extended, nonminimal Higgs sector can also significantly (and perhaps more naturally) enhance the first-order character of the EW phase transition [27,28]. We thus do not regard the requirement of a light stop as a necessary feature of a supersymmetric setup giving successful EWB. From the viewpoint of EDMs, we considered and evaluated the size of the two-loop stop-mediated contributions in [17], and concluded that they are subdominant, provided the left-handed stop is heavy enough, as required for EWB in the MSSM. ${ }^{4}$ In the interest of singling out the two-loop Higgs-mediated chargino-neutralino contributions under investigation here, and in view of the above considerations, we do not assume a light right-handed stop.

Having these considerations in mind, we illustrate in Fig. 4 the values of the CPV phase giving rise to an electron (left) and neutron (right) EDM equal to the current experi-

\footnotetext{
${ }^{4}$ Note that this requirement stems primarily from having a Higgs mass consistent with the LEP limits
}

mental limit, in the $\left(M_{1}, \mu\right)$ plane. We assume a minimal supergravity-type relation ${ }^{5}$ between the gaugino soft breaking masses (where gaugino masses unify at the GUT scale, and their EW-scale values are set by renormalization group running), and set here $M_{2}=2 M_{1}$. In addition, $\tan \beta$ and $m_{A^{0}}$ are set to the reference values listed in Eq. (11). In the low $\mu$ and low $M_{1}$ region the size of the two-loop contribution exceeds the experimentally viable values (which we take to be $d_{e}<1.6 \times 10^{-27} e \mathrm{~cm}$ and $d_{e}<$ $\left.2.9 \times 10^{-26} e \mathrm{~cm}\right)$ for a maximal CPV phase. For each value of the CPV phase, we indicate the boundaries of the excluded region. The parameter space above the various lines is currently experimentally open even for $\sin \phi_{\mu}=1$.

Depending on the supersymmetry breaking mechanism, gaugino masses can be related by different functional forms. For instance, if supersymmetry breaking is dominated by anomaly mediation, then one expects $M_{1} \simeq 3 M_{2}$, hence an inverted $b$-ino- $W$-ino hierarchy with respect to a gaugino-unified setup (see e.g. [29]). We study this possibility in Fig. 5, where we explore the values of the electron (left) and neutron (right) EDM on the $\left(M_{2}, \mu\right)$ plane, setting $M_{1}=3 M_{2}$ and the other parameters as in the benchmark model. We observe a pattern for the EDM

\footnotetext{
5Notice that the label "[mSUGRA]" obviously does not refer to the usual minimal supergravity setup, but only to the gaugino mass relation being employed here. The same applies to the label "[mAMSB]" of Fig. 5.
} 
Electron EDM $-\sin \phi_{\mu}=1, M_{A}=300 \mathrm{GeV}, M_{1}=3 M_{2}[\mathrm{mAMSB}]$

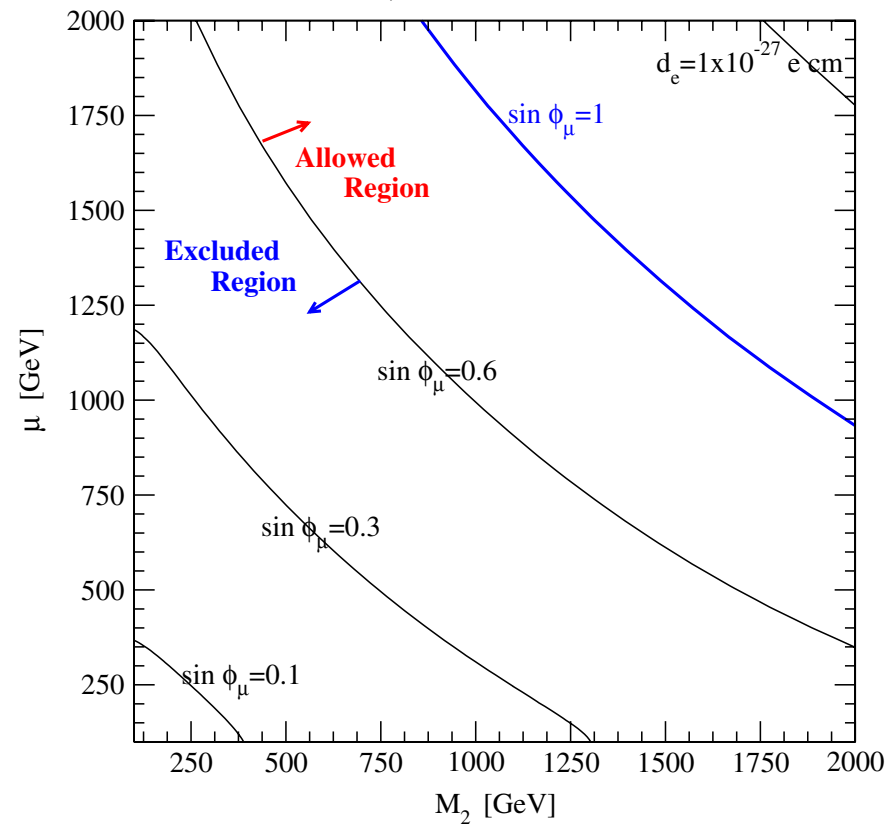

Neutron EDM $-\sin \phi_{\mu}=1, M_{A}=300 \mathrm{GeV}, M_{1}=3 M_{2}[m A M S B]$

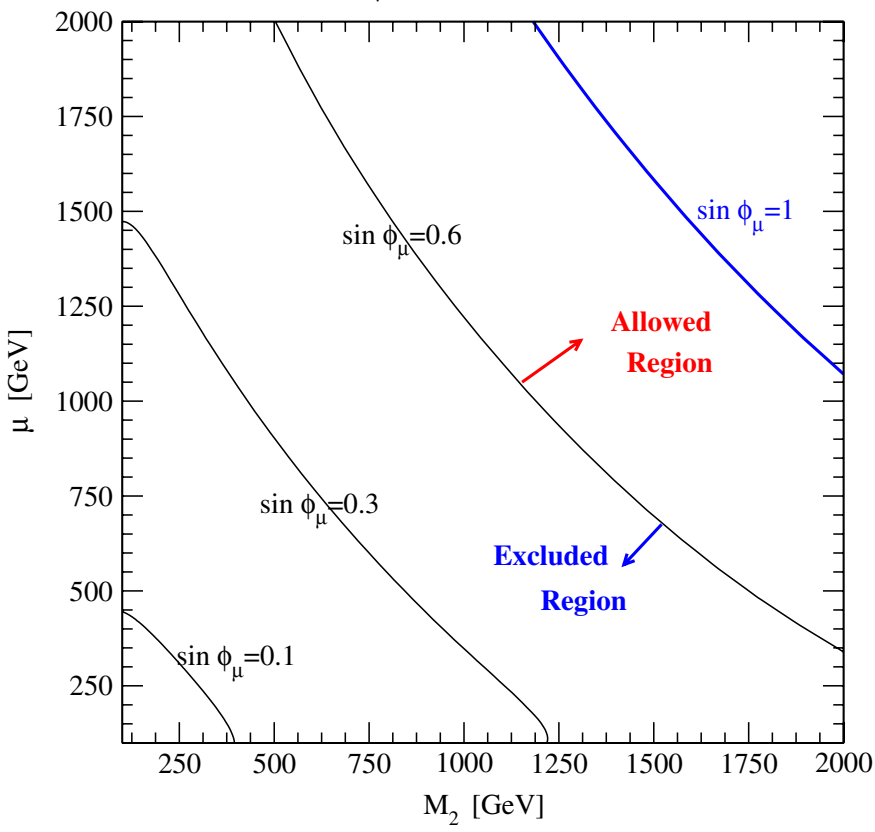

FIG. 5 (color online). Exclusion limits for the electron (left) and neutron (right) electric dipole moment, on the $\left(M_{2}, \mu\right)$ plane. We assume here the anomaly-mediated SUSY breaking gaugino mass relation $M_{1} \simeq 3 M_{2}$. In the upper right corner of the left panel we also show the contour of electron EDM equal to $10^{-27} e \mathrm{~cm}$, for maximal CPV phase.

very much similar to the one obtained for the gauginounification type scenario.

In passing, we note that a misalignment of the relative CPV phase between $M_{1}$ and $M_{2}$ does not affect our numerical results. In particular, we find that the main driver for the 2-loop EDM we consider here is the relative phase between $M_{2}$ and $\mu$, while a negligible contribution originates from the relative phase between $M_{1}$ and $\mu$. This result can potentially have profound implications for the interplay between electroweak baryogenesis and EDM searches, which we plan to explore in a future study.

In summary, our numerical results indicate that the new contributions computed here are dominant for the electron and neutron EDM at the two-loop level (in the limit of heavy sfermions) and should thus be included in any study of EDMs and $C P$ violation in the MSSM.

\section{SUMMARY AND CONCLUSIONS}

The analysis we have completed gives the EDMs of the electron and neutron in the MSSM when the sfermion masses are large, leading to a suppression of the oneloop contributions and general dominance of two-loop terms. Considering this regime allows one to circumvent the SUSY $C P$ problem associated with the present, stringent EDM limits and sub-TeV scale sfermions. It also implies vanishing EDMs for diatomic atoms, assuming they are generated primarily by the chromo-EDMs of the quarks. ${ }^{6}$ Previous studies of this regime have generally also taken all but the lightest, SM-like $C P$-even scalar to be heavy, thereby suppressing two-loop contributions involving the other Higgs scalars as well. In the present study, we have not made these assumptions and have, instead, analyzed the dependence of the two-loop EDMs on the full gauge-gaugino-Higgs-Higgsino parameter space of the MSSM.

Our primary result is that contributions arising from exchanges involving one SM gauge boson and either the $C P$-odd neutral scalar, $A^{0}$, or the charged Higgs scalars, $H^{ \pm}$, are comparable or dominant to previously considered contributions. We have also analyzed the prospective implications for MSSM electroweak baryogenesis, whose viability depends in part on the values of $\tan \beta$ and $m_{A^{0}}$. We leave a thorough exploration of the MSSM EWB to a more comprehensive future study [20].

\section{ACKNOWLEDGMENTS}

We thank G. Giuidice and A. Romanino for helpful discussions regarding the results for the $Z h^{0}$ contributions. This work was supported in part under U.S. Department of Energy Contract No. DE-FG02-08ER41531 (Y.L. and M.J.R.M.) and the University of Wisconsin Alumni

\footnotetext{
${ }^{6}$ See Ref. [4] for a detailed discussion of the effective operatordependence of various EDMs.
} 
Research Foundation (Y.L. and M.J.R.M.), and by a Faculty Research Grant from the University of California, Santa Cruz (S. P.).

\section{APPENDIX A: COEFFICIENTS, MATRICES, AND LOOP FUNCTION}

The coefficients $c_{u, d, e}^{h^{0}, H^{0}, A^{0}, H^{+}}$depend on specific types of Higgs bosons and SM fermions

$$
\begin{gathered}
c_{u}^{h^{0}}=\frac{Z_{R}^{21}}{\sin \beta}, \quad c_{d}^{h^{0}}=c_{e}^{h^{0}}=\frac{Z_{R}^{11}}{\cos \beta}, \quad c_{u}^{H^{0}}=\frac{Z_{R}^{22}}{\sin \beta}, \\
c_{d}^{H^{0}}=c_{e}^{H^{0}}=\frac{Z_{R}^{12}}{\cos \beta}, \quad c_{u}^{A^{0}}=\cot \beta, \quad c_{d}^{A^{0}}=c_{e}^{A^{0}}=\tan \beta, \\
c_{u}^{H^{+}}=\cot \beta, \quad c_{d}^{H^{+}}=c_{e}^{H^{+}}=\tan \beta .
\end{gathered}
$$

The matrices $D_{h^{0}, H^{0}}^{R, L}, G^{R, L}, E^{R, L}, M^{R, L}, N^{R, L}$ are

$$
\begin{gathered}
D_{h^{0}, a b}^{R}=Z_{R}^{11} Z_{-}^{2 b *} Z_{+}^{1 a *}+Z_{R}^{21} Z_{-}^{1 b *} Z_{+}^{2 a *}, \quad D_{h^{0}, a b}^{L}=Z_{R}^{11} Z_{-}^{2 a} Z_{+}^{1 b}+Z_{R}^{21} Z_{-}^{1 a} Z_{+}^{2 b}, \quad D_{H^{0}, a b}^{R}=Z_{R}^{12} Z_{-}^{2 b *} Z_{+}^{1 a *}+Z_{R}^{22} Z_{-}^{1 b *} Z_{+}^{2 a *} \\
D_{H^{0}, a b}^{L}=Z_{R}^{12} Z_{-}^{2 a} Z_{+}^{1 b}+Z_{R}^{22} Z_{-}^{1 a} Z_{+}^{2 b}, \quad G_{a b}^{R}=\frac{1}{2}\left(Z_{-}^{1 a} Z_{-}^{1 b *}+\delta^{a b}\left(c_{W}^{2}-s_{W}^{2}\right)\right), \quad G_{a b}^{L}=\frac{1}{2}\left(Z_{+}^{1 a *} Z_{+}^{1 b}+\delta^{a b}\left(c_{W}^{2}-s_{W}^{2}\right)\right) \\
E_{a b}^{R}=\sin \beta Z_{-}^{2 b *} Z_{+}^{1 a^{*}}+\cos \beta Z_{-}^{1 b *} Z_{+}^{2 a *}, \quad E_{a b}^{L}=-\left(\sin \beta Z_{-}^{2 a} Z_{+}^{1 b}+\cos \beta Z_{-}^{1 a} Z_{+}^{2 b}\right), \quad M_{a i}^{R}=Z_{-}^{1 a} Z_{N}^{2 i *}+\frac{1}{\sqrt{2}} Z_{-}^{2 a} Z_{N}^{3 i *} \\
M_{a i}^{L}=Z_{+}^{1 a *} Z_{N}^{2 i}-\frac{1}{\sqrt{2}} Z_{+}^{2 a *} Z_{N}^{4 i}, \quad N_{a i}^{R}=-\cos \beta\left(\frac{1}{\sqrt{2}} Z_{+}^{2 a *}\left(Z_{N}^{1 i *} s_{W}+Z_{N}^{2 i *} c_{W}\right)+Z_{+}^{1 a *} Z_{N}^{4 i *} c_{W}\right) \\
N_{a i}^{L}=\sin \beta\left(\frac{1}{\sqrt{2}} Z_{-}^{2 a}\left(Z_{N}^{1 i} s_{W}+Z_{N}^{2 i} c_{W}\right)-Z_{-}^{1 a} Z_{N}^{3 i} c_{W}\right)
\end{gathered}
$$

with $\tan \beta=v_{u} / v_{d}$.

The $Z_{ \pm, N}$ are diagonalization matrices of chargino and neutralino mass matrices $Z_{-}^{T} M_{C} Z_{+}=\operatorname{Diag}\left(M_{\chi_{1}^{+}}, M_{\chi_{2}^{+}}\right)$, $Z_{N}^{T} M_{N} Z_{N}=\operatorname{Diag}\left(M_{\chi_{1}^{0}}, M_{\chi_{2}^{0}}, M_{\chi_{3}^{0}}, M_{\chi_{4}^{0}}\right)$, with $M_{\chi_{a}^{+}}>0, M_{\chi_{i}^{0}}>0$, and

$$
M_{C}=\left(\begin{array}{cc}
M_{2} & \sqrt{2} M_{W} \sin \beta \\
\sqrt{2} M_{W} \cos \beta & \mu
\end{array}\right), \quad M_{N}=\left(\begin{array}{cccc}
M_{1} & 0 & -M_{Z} s_{W} \cos \beta & M_{Z} s_{W} \sin \beta \\
0 & M_{2} & M_{Z} c_{W} \cos \beta & -M_{Z} c_{W} \sin \beta \\
-M_{Z} s_{W} \cos \beta & M_{Z} c_{W} \cos \beta & 0 & -\mu \\
M_{Z} s_{W} \sin \beta & -M_{Z} c_{W} \sin \beta & -\mu & 0
\end{array}\right) .
$$

The $Z_{R}$ in $c^{h^{0}, H^{0}}$ and $D_{h^{0}, H^{0}}^{R, L}$ is the matrix that diagonalize the mass matrix of $C P$-even neutral Higgs bosons

$$
\sqrt{2}\left(\begin{array}{l}
\operatorname{Re}\left[H_{d}^{0}\right]-v_{d} \\
\operatorname{Re}\left[H_{u}^{0}\right]-v_{u}
\end{array}\right)=Z_{R}\left(\begin{array}{c}
h^{0} \\
H^{0}
\end{array}\right)
$$

which, expressed in terms of $\alpha$, takes the form

$$
Z_{R}=\left(\begin{array}{cc}
-\sin \alpha & \cos \alpha \\
\cos \alpha & \sin \alpha
\end{array}\right)
$$

The $\alpha$, at tree level, can be expressed as

$$
\tan \alpha=\frac{-\left(M_{A^{0}}^{2}-M_{Z}^{2}\right) \cos 2 \beta-\sqrt{\left(M_{A^{0}}^{2}+M_{Z}^{2}\right)^{2}-4 M_{A^{0}}^{2} M_{Z}^{2} \cos ^{2} 2 \beta}}{\left(M_{A^{0}}^{2}+M_{Z}^{2}\right) \sin 2 \beta} .
$$

Notice that $\alpha$ is approximately $\beta-\pi / 2$ at the limit $M_{A^{0}} \gg M_{Z}$. Radiative corrections modify the latter relation, and we include these effects in our numerical study.

The loop function $j\left(r, r^{\prime}\right)$ is the same as in Ref. [11]

$$
j(r)=\frac{r \log r}{r-1}, \quad j\left(r, r^{\prime}\right)=\frac{j(r)-j\left(r^{\prime}\right)}{r-r^{\prime}} .
$$


[1] B.C. Regan, E.D. Commins, C. J. Schmidt, and D. DeMille, Phys. Rev. Lett. 88, 071805 (2002).

[2] C. A. Baker et al., Phys. Rev. Lett. 97, 131801 (2006).

[3] M. V. Romalis, W. C. Griffith, and E. N. Fortson, Phys. Rev. Lett. 86, 2505 (2001).

[4] M. Pospelov and A. Ritz, Ann. Phys. (N.Y.) 318, 119 (2005).

[5] M. J. Ramsey-Musolf and S. Su, Phys. Rep. 456, 1 (2008).

[6] A. G. Cohen, D. B. Kaplan, and A. E. Nelson, Phys. Lett. B 388, 588 (1996).

[7] D. Chang, W. Y. Keung, and A. Pilaftsis, Phys. Rev. Lett. 82, 900 (1999)83, 3972(E) (1999); A. Pilaftsis, Phys. Lett. B 471, 174 (1999).

[8] D. Chang, W. F. Chang, and W. Y. Keung, Phys. Lett. B 478, 239 (2000).

[9] D. Chang, W. F. Chang, and W. Y. Keung, Phys. Rev. D 66, 116008 (2002).

[10] A. Pilaftsis, Nucl. Phys. B644, 263 (2002).

[11] G. F. Giudice and A. Romanino, Phys. Lett. B 634, 307 (2006).

[12] D. Chang, W. F. Chang, and W. Y. Keung, Phys. Rev. D 71, 076006 (2005).

[13] S. M. Barr, and A. Zee, Phys. Rev. Lett. 65, 21 (1990); 65, 2920(E) (1990).

[14] T. F. Feng, L. Sun, and X. Y. Yang, Nucl. Phys. B800, 221 (2008).

[15] K. Fujikawa, Phys. Rev. D 7, 393 (1973); M. B. Gavela, G. Girardi, C. Malleville, and P. Sorba, Nucl. Phys. B193, 257 (1981); J. C. Romao and A. Barroso, Phys. Rev. D 35, 2836 (1987).

[16] J. Rosiek, arXiv:hep-ph/9511250.

[17] V. Cirigliano, S. Profumo, and M. J. Ramsey-Musolf, J.
High Energy Phys. 07 (2006) 002.

[18] S. Heinemeyer, W. Hollik, and G. Weiglein, Comput. Phys. Commun. 124, 76 (2000).

[19] E. Komatsu et al. (WMAP Collaboration), arXiv:0803.0547.

[20] V. Cirigliano, Y.C. Li, S. Profumo, and M. J. RamseyMusolf (work in progress).

[21] W. M. Yao et al. (Particle Data Group), J. Phys. G 33, 1 (2006).

[22] J. Ellis, J. S. Lee, and A. Pilaftsis, arXiv:0808.1819.

[23] T. Ibrahim and P. Nath, Phys. Rev. D 57, 478 (1998); 58, 019901(E) (1998); 60, 079903(E) (1999); 60, 119901(E) (1999).

[24] J. R. Ellis and R. A. Flores, Phys. Lett. B 377, 83 (1996).

[25] J. M. Moreno, M. Quiros, and M. Seco, Nucl. Phys. B526, 489 (1998).

[26] M. S. Carena, M. Quiros, M. Seco, and C. E. M. Wagner, Nucl. Phys. B650, 24 (2003).

[27] M. Pietroni, Nucl. Phys. B402, 27 (1993); A. T. Davies, C. D. Froggatt, and R. G. Moorhouse, Phys. Lett. B 372, 88 (1996); S. J. Huber and M. G. Schmidt, Nucl. Phys. B606, 183 (2001); M. Bastero-Gil, C. Hugonie, S. F. King, D. P. Roy, and S. Vempati, Phys. Lett. B 489, 359 (2000); J. Kang, P. Langacker, T. j. Li, and T. Liu, Phys. Rev. Lett. 94, 061801 (2005); A. Menon, D. E. Morrissey, and C. E. M. Wagner, Phys. Rev. D 70, 035005 (2004); C. Grojean, G. Servant, and J.D. Wells, Phys. Rev. D 71, 036001 (2005); M. Carena, A. Megevand, M. Quiros, and C. E. M. Wagner, Nucl. Phys. B716, 319 (2005).

[28] S. Profumo, M. J. Ramsey-Musolf, and G. Shaughnessy, J. High Energy Phys. 08 (2007) 010.

[29] T. Moroi and L. Randall, Nucl. Phys. B570, 455 (2000). 